(mean $4.7 \mathrm{mmol} / \mathrm{mol}$, and results are pending for 18 patients.Collection of outcomes including hospitalisations for $\mathrm{HF}, \mathrm{CV}$ events, ejection fraction, and adverse effects of treatment is ongoing. The above has been achieved despite limitations imposed by the remote nature of the clinic due to the Covid-19 pandemic, which limits performance of blood tests, echocardiograms and observations. This limitation is expected to be ameliorated by conducting in-person clinics in future.

Conclusions Operation of a joint CMC facilitates optimisation of the pharmacological management of risk factors in patients with cardiac and metabolic disease, particularly incorporation of current evidence-based therapies. Emerging outcomes indicate the potential impact of this service on patients' long term CV outcomes.

Conflict of Interest Nil

\section{PRIMARY CARE USE OF SGLT2 INHIBITORS IN PATIENTS WITH TYPE 2 DIABETES MELLITUS AND CARDIOVASCULAR DISEASE - ARE WE MISSING A TRICK?}

${ }^{1}$ David Higson, ${ }^{2}$ Andrew Turley. ${ }^{1}$ Hull York Medical School, York, UK; ${ }^{2}$ South Tees Hospitals NHS Foundation Trust

\subsection{6/heartjnl-2021-BCS.194}

Background Patients with Type 2 Diabetes Mellitus (T2DM) and Atherosclerotic Cardiovascular Disease (ASCVD) have an average reduction of 12 years in life expectancy. The landmark EMPA-REG trial in 2013 demonstrated that SGLT2i significantly reduced risk of all-cause mortality in this population of patients. The NNT was 39 at 3.1 years. This is comparable to other landmark studies such as the $4 \mathrm{~S}$ study (Simvastatin NNT 30 at 5.3 years) and the HOPE trial (Ramipril, NNT 50 at 5 years). International guidelines recognise the CV benefits of SGLT2i. They suggest SGLT2i should be used as a second line therapy or as a first line intervention in treatment naïve T2DM patients who have ASCVD.

Objectives To assess use of SGLT2 $\mathrm{i}$ in patients with T2DM and known ASCVD in primary care. Additionally, to understand the potential benefit of introducing SGLT2 $i$ to these patients in primary care.

Methods The patient list of a GP surgery was searched for patients coded to have T2DM and ASCVD. Demographics, most recent $\mathrm{HbA} 1 \mathrm{c}$ and eGFR, diabetic and cardiology medication were recorded. Search criteria were as follows: Myocardial Infarction, Unstable Angina, Ischaemic Stroke, Haemorrhagic Stroke, Peripheral Artery Disease, AAA,

\section{Patients on medication with CV benefit}

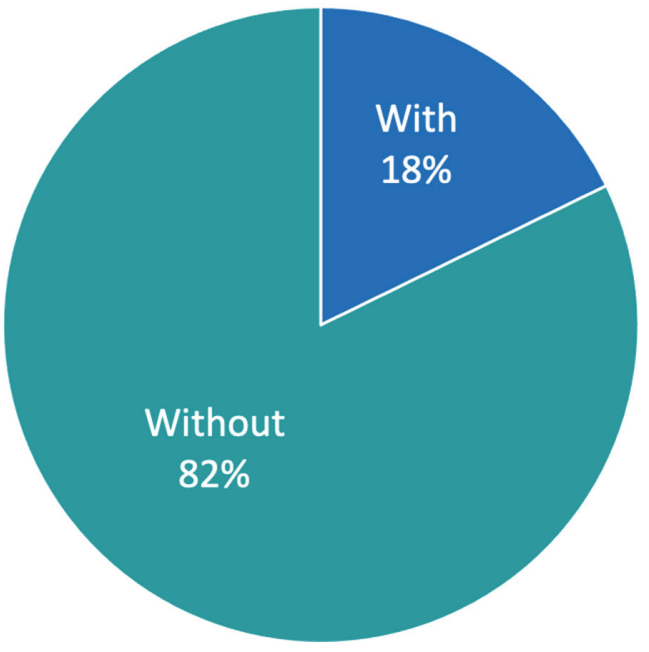

Abstract 198 Figure 1

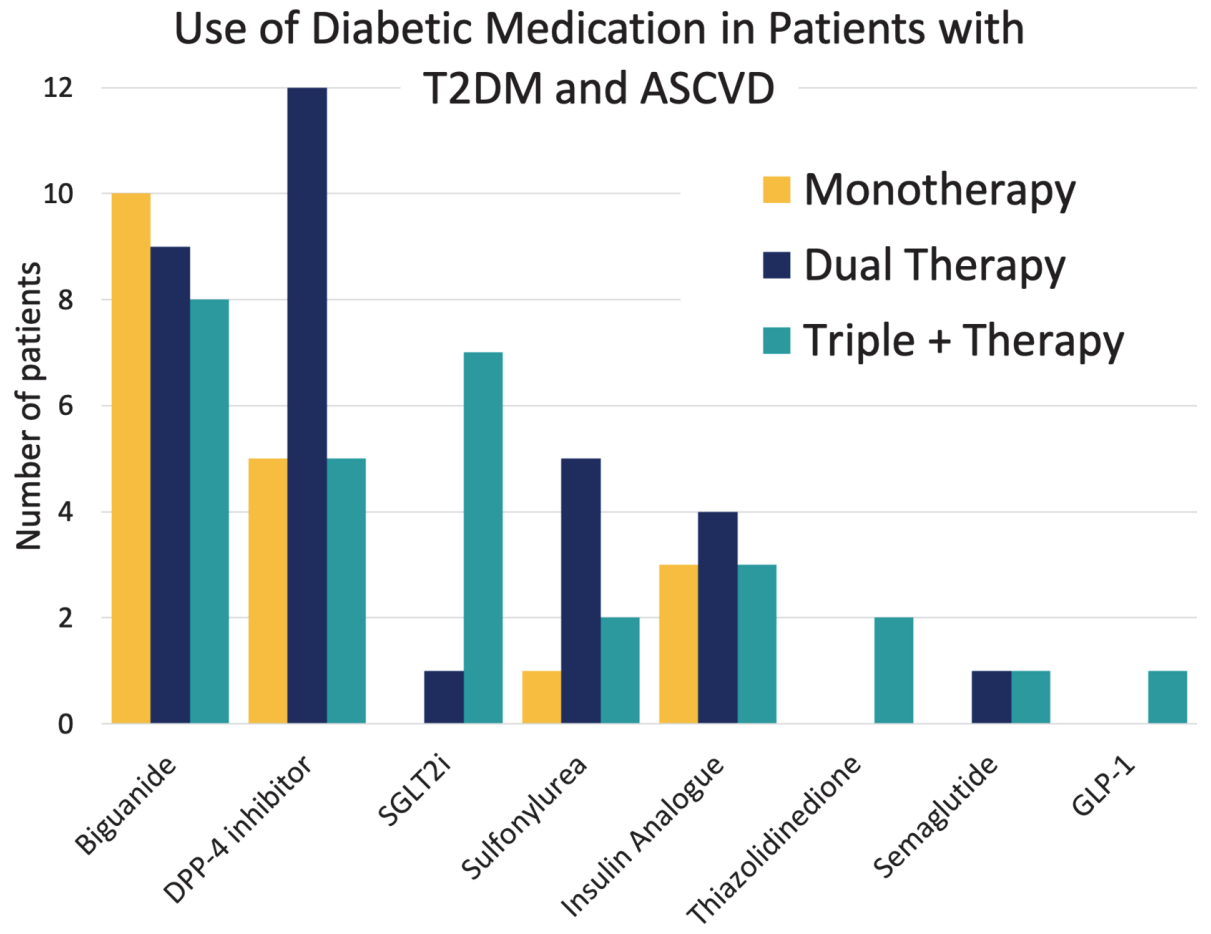


Coronary Artery Bypass Graft, Percutaneous Coronary Intervention, Percutaneous Transluminal Coronary Angioplasty.

Results 8525 patients registered, 487 had T2DM (5.7\%) and 68 patients were identified as having T2DM and ASCVD. 5 were excluded from analysis, one patient had left the surgery, three had no coronary atheroma on angiogram and one was mis-coded. Therefore 63 patients were used for analysis (12.9\% of those with T2DM). Mean age 77.2 (SD 10.13), 41 male and 22 female. 58 patients had an eGFR >30, 29 patients had an eGFR $>60.45$ patients were receiving at least one medication for T2DM (table 1). 18\% of patients taking medication were receiving either GLP-1 or SGLT2i which confer CV benefit (figure 1). $75 \%$ of patients on dual therapy were taking DPP-4 inhibitors compared with $6.25 \%$ patients who were taking SGLT2i. DPP-4 inhibitors have no CV benefit. SGLT2i were typically used as a third line or later therapy (figure 2).

Conclusion SGLT2i were used sparingly and typically as a third line therapy or later in this cohort. DPP-4 inhibitors tended to be used as second line but lack any $\mathrm{CV}$ benefits. With a NNT of 39, concerted efforts to increase SGLT2i use in patients with T2DM and ASCVD have the potential to be an effective intervention in primary care that ultimately saves lives.

Conflict of Interest None

\section{AUTONOMIC FUNCTION IN RESISTANT AND MALIGNANT HYPERTENSION}

${ }^{1}$ Reem Alsharari, ${ }^{2}$ Neil Thomas, ${ }^{3} \mathrm{G}$. Lip, ${ }^{3}$ Alena Shantsila. ${ }^{1}$ Institute of Cardiovascular Sciences, University of Birmingham, Birmingham, UK; ${ }^{2}$ Institute of Applied Health Research, University of Birmingham; ${ }^{3}$ Liverpool Centre for Cardiovascular Science, University of Liverpool and Liverpool Heart \& Chest

\subsection{6/heartjnl-2021-BCS.195}

Background Enhanced sympathetic activity and reduced parasympathetic activity, assessed by heart rate variability (HRV) indices, have been linked to the pathogenesis of hypertension. Some studies showed that sympathetic and parasympathetic activity tend to restore after long-term hypertension exposure, as a result of cardiac output adaptation. It has not been known whether resistant hypertension (RH) and treated malignant hypertension (MHT) patients experienced similar restoration of autonomic balance.

Purpose To explore the autonomic changes in treated MHT, $\mathrm{RH}$ and normotensives subjects.

Methods We studied 23 patients with RH (57 \pm 11 y), 18 patients with treated MHT $(54 \pm 13 \mathrm{y})$, and 23 normotensives controls (NC) $(50 \pm 5 \mathrm{y})$. Time domain and frequency domain HRV indices of 5 minutes recordings were used to evaluate autonomic function. In the time domain, standard deviation of normal-to-normal R-R intervals (SDNN) reflect parasympathetic activity. Reduced SDNN is a marker of lower parasympathetic tone. The ratio between low frequency and high frequency spectrum was assessed in frequency domain. LF/HF increased is a marker of increased sympathetic activity.

Results The groups were matched by age and body surface area (all $\mathrm{p}>0.05)$. Time domain and frequency domain variables of HRV were not significantly different between three groups $(p>0.05$ for all) (table 1). Antihypertensive
Abstract 199 Table 1 Demographic and cardiovascular parameters

\begin{tabular}{lllll}
\hline & NC(n=23) & MHT(n=18) & RH(n=23) & $\begin{array}{l}\text { P- } \\
\text { value }\end{array}$ \\
\hline Office SBP (mmHg) & 1207 & $16632^{*}$ & $16321 \dagger$ & $<0.001$ \\
Office DBP (mmHg) & 788 & $9717^{*}$ & $9516 \dagger$ & $<0.001$ \\
Duration of hypertension & $0[0-0]$ & $7[5-8]^{*}$ & $8[5-10] \dagger$ & $<0.001$ \\
(years) & & & & \\
SDNN (ms) & $48[30-81]$ & $47[34-64]$ & $48[25-68]$ & 0.89 \\
rMSSD (ms) & $33[25-51]$ & $30[23-50]$ & $27[16-40]$ & 0.26 \\
pNN50 (\%) & $9[3-30]$ & $6[3-18]$ & $6[1-17]$ & 0.39 \\
LF (ms2) & $809[198-$ & $642[276-$ & $398[186-$ & 0.39 \\
& $1849]$ & $955]$ & $935]$ & \\
HF (ms2) & $314[185-789]$ & $327[128-$ & $203[92-481]$ & 0.49 \\
& & $366]$ & & \\
HF (n.u) & 3418.3 & 33.116 & 3622 & 0.37 \\
LF (n.u) & 6618.3 & 6715.7 & 6422 & 0.91 \\
LF/HF & 1.90 .4 & 2.020 .3 & 1.770 .5 & 0.97 \\
RSA & 143.2 & 143.2 & 143 & 0.89 \\
\hline
\end{tabular}

Normally distributed data are expressed as (mean \pm SD). Non-normally distributed data are displayed as median with interquartile ranges. ${ }^{*} P<0.05$ between healthy controls and malignant hypertension. $\mathrm{TP}<0.05$ between healthy controls and resistant hypertension. DBP: diastolic blood pressure; HF: High frequency spectrum; LF: Low frequency spectrum; MHT: Malignant hypertension; NC: Normotensives control; pNN50: Percentage of successive differences between R-R intervals greater than $50 \mathrm{~ms}$; RH: Resistant hypertension; rMSSD: Square root of the mean of the successive differences between adjacent R-R intervals; RSA respiratory rate; SBP: systolic blood pressure; SDNN: Standard deviation of normal to normal R-R intervals.

RH was defined as office systolic and diastolic blood pressures exceeds 140/90 mmHg despite the use of three or more antihypertensive medications, one of which is a diuretic. MHT was defined as a diastolic blood pressure of $120 \mathrm{mmHg}$ or more, accompanied by bilateral retinal haemorrhages and/or exudates, with or without papilledema.

medications used were similar, except for the higher use of diuretics in RH group $(100 \%$ vs. $67 \%, \mathrm{p}<0.05)$. On linear regression, independent predictors of decreased SDNN were high creatinine level, decreased subendocardial viability ratio and increased central systolic blood pressure $(\mathrm{p}<0.05)$. On linear regression, independent predictor of high $\mathrm{HF} / \mathrm{LF}$ ratio was presence of left ventricular hypertrophy $(\beta=-2.6$, $\mathrm{p}=0.04)$.

Conclusion No differences were detected in HRV parameters between groups. These findings support the hypothesis of potential restoration of sympatho-vagal balance after prolonged hypertension exposure. Presence of target organ damage independently predicts decreased parasympathetic and increased sympathetic tone.

Conflict of Interest None

\section{ASSOCIATIONS OF TRIGLYCERIDE LEVEL AND VARIABILITIES WITH LUNG RELATED INFECTIONS, CANCER, AND MORTALITY OUTCOMES: A TERRITORY- WIDE COHORT STUDY}

${ }^{1}$ Keith Sai Kit Leung, ${ }^{2}$ Gary Tse, ${ }^{3}$ Jiandong Zhou, ${ }^{4}$ Ishan Lakhani, ${ }^{4}$ Sharen Lee, ${ }^{3}$ Qingpeng Zhang, ${ }^{2}$ Tong Liu. ${ }^{1}$ Aston Medical School, Birmingham, UK; ${ }^{2}$ Tianjin Institute of Cardiology, Second Hospital of Tianjin Medical University; ${ }^{3}$ School of Data Science, City University of Hong Kong; ${ }^{4}$ Cardiovascular Analytics Group, Laboratory of Cardiovascular Physiology, Hong Kong

10.1136/heartjnl-2021-BCS.196 